\title{
Maltrato infantil: conocimientos, actitudes y prácticas de estudiantes de Odontología de Cartagena, Colombia
}

\author{
Katherine M. Arrieta Vergaraa ${ }^{a}$, Shyrley Díaz Cárdenas ${ }^{\mathrm{b}}$ y Farith González Martínez ${ }^{\mathrm{c}}$
}

\author{
a Odontóloga. Magíster en \\ Salud Pública. Especialista \\ en Gerencia en Salud. \\ Departamento de Odontología \\ Preventiva y Social. \\ Facultad de Odontología. \\ Universidad de Cartagena. \\ Cartagena de Indias \\ (Colombia). \\ b Odontóloga. Magíster en \\ Salud Pública. Especialista \\ en Gerencia en Salud y Salud \\ Familiar. Departamento de \\ Odontología Preventiva \\ y Social. Facultad de \\ Odontología. Universidad \\ de Cartagena. Cartagena de \\ Indias (Colombia). \\ c Odontólogo. Magíster en \\ Salud Pública. Especialista \\ en Investigación Social. \\ Jefe del Departamento de \\ Investigaciones. Facultad de \\ Odontología. Universidad \\ de Cartagena. Cartagena de \\ Indias (Colombia).
}

Correspondencia: Katherine M. Arrieta Vergara. Departamento de Odontología Preventiva y Social. Facultad de Odontología. Campus de la Salud. Universidad de Cartagena. Barrio Zaragocilla. Cartagena de Indias

(Colombia).

Correo electrónico: katherinearrieta@gmail.com

Recibido el 17 de marzo de 2014.

Aceptado para su publicación el 1 de abril de 2014.

\author{
RESUMEN
}

Objetivo: Describir los conocimientos, actitudes y prácticas sobre maltrato infantil en estudiantes de odontología en una universidad pública de Cartagena, Colombia.

Diseño del estudio: Estudio descriptivo.

Emplazamiento: Universidad pública de Cartagena, Colombia.

Participantes: 208 estudiantes de odontología de Cartagena (Colombia), seleccionados a través de un muestreo probabilístico.

Mediciones principales: Se diseñó y utilizó un instrumento tipo cuestionario estructurado anónimo de auto-reporte para evaluar las variables: sociodemográficas, conocimientos (relacionados con maltrato físico, abuso sexual, negligencia, maltrato psicológico, indicadores de maltrato infantil y responsabilidades), actitudes, prácticas y prevalencia de posibles sospechas de casos de maltrato infantil detectados en la práctica odontológica. Los datos fueron analizados a través de distribuciones de frecuencia y proporciones. Para establecer relaciones entre la sospecha de maltrato infantil con algunas variables de interés se utilizó la prueba $\chi^{2}$ asumiendo un límite de 0,05 para la significación.

Resultados: La prevalencia actual de casos de sospecha de maltrato infantil fue del 2,4 \%, y la de alguna vez del $20 \%$. Los conocimientos fueron buenos para el $54,3 \%$ de los sujetos y aceptable en el 44,7 \%; las actitudes favorables en el 96,1\%. De 42 estudiantes que tuvieron alguna sospecha durante su práctica, 15 manifestaron como primera acción comunicarla a su docente, seguida por comentarla a un compañero y guardar silencio. Las tres cuartas partes de los que manifestaron haber tenido una sospecha de maltrato no la consignaron en la historia clínica.

Conclusiones: La prevalencia de posibles casos de maltrato infantil identificados por los estudiantes de odontología fue alta; sus conocimientos y actitudes fueron adecuados. Sin embargo, existe incongruencia con las prácticas reportadas; por esto es necesario realizar entrenamientos en la temática que brinden una mayor seguridad al estudiante en el momento de identificar y reportar los posibles casos de maltrato infantil desde la práctica odontológica.

Palabras clave: Estudiantes de Odontología. Conocimientos, Actitudes y Práctica en Salud. Maltrato a los Niños.

\begin{abstract}
Child abuse: knowledge, attitudes and practices of dental students in Cartagena, Colombia

Objetive: to describe the knowledge, attitudes and practices in dental students at the Cartagena state university, Colombia regarding child abuse.

Study design: descriptive study.

Location: Cartagena state university, Colombia.

Participants: 208 dental students from Cartagena (Colombia), selected through probability sampling.

Main analysed data: A self-reporting anonymous structured questionnaire-type instrument was designed and used to evaluate socio-demographic variables, knowledge (related to physical abuse, sexual abuse, negligence, psychological abuse, signs of child abuse and responsibilities), attitudes, practices and prevalence of possible suspicion of cases of child abuse detected in dental practice). The data were analysed through frequency distributions and proportions. In order to determine relationships between suspicion of child abuse and some variables of interest, test $\mathrm{c}^{2}$ was used, assuming a limit of 0.05 for significance.

Results: Current prevalence of suspected cases of child abuse was $2.4 \%$ and that of it occurring at sometime, $20 \%$. Knowledge was good for $54.3 \%$ of the subjects and acceptable for $44.7 \%$; favourable attitudes, $96.1 \%$. Of 42 students who had some kind of suspicion during their practice, 15 stated that their first action was to report it to their teacher, followed by telling a classmate and keeping quiet. Three quarters of those who stated they had suspected abuse did not enter it in the clinical record.

Conclusions: The prevalence of possible cases of child abuse identified by dental students was high; their knowledge and attitudes were appropriate. However, there is incongruence in reported practices; for this reason, it is necessary to carry out training on the subject to provide the student with greater certainty on identifying and reporting possible cases of child abuse from the position of dental practice.
\end{abstract}

Key words: Students, Dental. Health Knowledge, Attitudes, Practice. Child abuse. 


\section{INTRODUCCIÓN}

El maltrato infantil (MI) se define según la OMS como "los abusos y la desatención de que son objeto los menores de 18 años de edad, e incluye todos los tipos de maltrato físico o psicológico, abuso sexual, desatención, negligencia y explotación comercial o de otro tipo que causen o puedan causar un daño a la salud, desarrollo o dignidad del niño"1. Éste incluye: lo que se hace (acción), lo que se deja de hacer (omisión) y lo que se realiza de forma inadecuada (negligencia) ${ }^{2}$.

En la literatura se describe una correlación entre el $\mathrm{Ml}$ y las lesiones patológicas en cabeza y cue$110^{3,4}$. Estudios epidemiológicos evidencian que del 50 al $77 \%$ de las lesiones físicas de niños maltratados aparecieron en la región oro-facial (boca, cara y cabeza $)^{5,6}$. Es por esto que el odontólogo o el estudiante de odontología durante sus prácticas clínicas, al tener cercanía con el paciente, cuenta una posición privilegiada para la identificación de los posibles casos de maltrato. Además, una deficiente salud oral a menudo se asocia también el abandono de los niños; a pesar de todo lo anterior el profesional de la odontología continua dejando de reportar estos casos $^{7-9}$.

Dentro de las barreras e inconvenientes que se han identificado para el reporte de Ml está la falta de conocimientos y el entrenamiento ${ }^{5,10,11}$; si un odontólogo es entrenado está más seguro de sus habilidades para reconocer el abuso y más casos pueden ser reportados y diagnosticados desde la consulta dental ${ }^{12}$.

Muchas profesiones del área de la salud no incluyen en sus planes de estudio y programas de educación continua aspectos de formación relacionados con el maltrato; por lo tanto, los profesionales egresados no están preparados para ofrecer una ayuda a las víctimas ${ }^{13}$. Es así que estudios realizados en varios países han demostrado que los conocimientos acerca de los problemas de protección de menores son deficientes ${ }^{14}$. Aldair et al. ${ }^{15}$ encontraron que la probabilidad del reporte de los odontólogos está relacionada con la exposición a la educación. De esta forma, la calidad de la educación odontológica es un factor importante en el incremento de la detección e identificación del MI.

Debido a los pocos estudios que existen en Colombia sobre los conocimientos, actitudes y prácticas frente al maltrato infantil en estudiantes de odontología se realiza este estudio, que tiene como objetivo describir la ocurrencia de sospecha de maltrato infantil y los conocimientos, actitudes y prácticas sobre maltrato infantil que poseen los estudiantes de odontología quienes, como futuros profesionales, deben reconocer a sus pacientes víctimas de abuso y darles el manejo adecuado a la situación haciendo los reportes del caso.

\section{SUJETOS Y MÉTODOS}

Estudio descriptivo, con enfoque cuantitativo, realizado en estudiantes de odontología de una universidad pública de la ciudad de Cartagena, Colombia, durante el segundo semestre de 2012. El tamaño de muestra fue calculado teniendo en cuenta una población universo de 599, un nivel de confianza del $95 \%$ y un error del $5 \%$, asumiendo una frecuencia esperada del 32 \% (notificación de maltrato infantil por los estudiantes de odontología $)^{16}$, obteniéndose un tamaño de muestra de 215. Los sujetos fueron seleccionados a través de un muestreo aleatorio simple, teniendo en cuenta los siguientes criterios: estar matriculado en semestres que incluyan actividades pre-clínicas o clínicas (sexto a décimo) y aceptar participar en el estudio a través de su consentimiento informado por escrito. La participación de los sujetos estuvo regulada por las normas éticas estipuladas en la declaración de Helsinki, modificación de Edimburgo 2000; y las normas científicas, técnicas y administrativas para la investigación en seres humanos, resolución 8430 de 1993 del Ministerio de salud de la República de Colombia.

Para la recolección de la información se diseñó y utilizó un cuestionario anónimo de auto-reporte que fue previamente evaluado en una prueba piloto, en la que se indagó sobre: la comprensión de las preguntas, suficiencias de las categorías, extensión de la encuesta y pertinencia de la misma. Incluyó preguntas cerradas, abiertas, y por selección de falso o verdadero, sobre: variables sociodemográficas (sexo, edad, semestre, estrato socioeconómico y procedencia), conocimientos relacionados con maltrato físico, abuso sexual, negligencia, maltrato psicológico, indicadores y diagnóstico de maltrato infantil y responsabilidades ante este evento. Estos conocimientos fueron evaluados en 24 preguntas, dándose 2 puntos a cada respuesta correcta; el nivel se estableció por rangos de escaso (0-24), aceptable (25-36) y bueno (37-48). De igual forma, se indagó sobre las actitudes y las prácticas de los estudiantes frente al maltrato infantil. Se evaluó el nivel de actitud por escala de Likert, dándose valores de 1 a 5 puntos a los ítems, con rangos de desfavorables (4-15) y favorables (16-20); y las prácticas en 4 preguntas de selección múltiple con 
única respuesta, además de preguntar por percepciones sobre la enseñanza recibida en el tema. Al evaluar la confiabilidad del instrumento se obtuvo un Alpha de Cronbach de 0,79 para conocimientos, 0,81 para actitudes y 0,78 para las prácticas.

Una vez identificados los sujetos se le explicaron los alcances y objetivo del estudio y se solicitó su participación a través de la firma de un consentimiento informado.

En cuanto al análisis e interpretación de los datos, se utilizaron pruebas de estadística descriptiva; se estimó la ocurrencia de sospecha de maltrato infantil a través de prevalencias y frecuencias y se determinaron los conocimientos, actitudes y prácticas frente al maltrato infantil. Los estimadores generados en consideración de los objetivos planteados en este estudio fueron calculados utilizando el programa Stata versión $10.0^{\circledR}$. Luego se realizaron cruces de variables para describir la distribución resto de las encuestas (7) quedaron fuera de análisis: 2 individuos no aceptaron participar y 5 fueron anuladas por mal cumplimentación.

La población estuvo compuesta por 133 mujeres $(63,9 \%)$ y 75 hombres $(30,1 \%)$. El $87 \%$ se encontraba en la etapa de adulto joven, donde la media de edad fue de 21,7 años (DE 2,11). En cuanto al semestre cursado, el $21,1 \%$ se encontraba en sexto semestre, el $13,9 \%$ en séptimo, el $25,4 \%$ en octavo, el $23,5 \%$ en noveno y el $15,8 \%$ en décimo.

\section{Sospecha de maltrato infantil}

Con respecto a la prevalencia actual de casos de sospecha de maltrato infantil se presentaron solo 5 sospechas $(2,4 \%)$, y en cuanto a la pregunta sobre sospecha de maltrato infantil alguna vez durante sus prácticas de formación como odontólogos se obtuvo una prevalencia del 20 \% (IC95 \%: 14,625,6) (tabla 1).

\begin{tabular}{lccc}
\hline Casos de sospecha de maltrato infantil & $\begin{array}{c}\text { Frecuencia } \\
(\mathbf{n = 2 0 8 )}\end{array}$ & $\begin{array}{c}\text { Porcentaje } \\
(\mathbf{\%})\end{array}$ & IC 95\% \\
\hline Sospecha actual de maltrato en sus pacientes & 5 & 2,4 & $0,3-4,5$ \\
Sí & 203 & 97,6 & $95,4-99,6$ \\
No & & & \\
Sospecha alguna vez de maltrato & 42 & 20,1 & $14,6-25,6$ \\
Sí & 166 & 79,8 & $74,3-85,3$ \\
No & & & \\
Número de sospechas durante sus prácticas & 26 & 12,5 & $7,9-17,0$ \\
1 vez & 13 & 6,25 & $2,9-9,5$ \\
2 veces & 3 & 1,44 & $0,1-3,0$ \\
3 veces & 166 & 79,8 & $74,3-85,3$ \\
No aplica & & & \\
\hline
\end{tabular}

Tabla 1. Prevalencia de casos de sospecha de maltrato infantil de estudiantes de Odontología de Cartagena, Colombia

de sospecha de maltrato infantil, en relación con algunas variables de interés (sociodemográficas, conocimientos y actitudes). Para establecer la significación del estimador en relación con otras variables se aplicó la prueba chi cuadrado $\left(\chi^{2}\right)$. Los estimadores se presentaran junto con sus intervalos de confianza al $95 \%$ y teniendo en cuenta un error máximo permitido del $5 \%$.

\section{RESULTADOS}

Para la presente investigación se tuvo un tamaño de la muestra de 215 estudiantes de sexto a décimo semestres de Odontología, de los cuales se obtuvieron 208 encuestas bien cumplimentadas; el

\section{Conocimientos}

Al evaluar el nivel de conocimientos sobre maltrato infantil en general se encontró que un poco más de la mitad de la población encuestada presentó buenos conocimientos en el tema (54,3\%; IC95 \% $47,5-61,1)$, seguido por el nivel aceptable $(44,7 \%$; IC95 \%: $37,89-51,52)$ y escaso $(0,9 \%$; IC $95 \%$ : 0,37-2,2).

De igual manera, se evaluaron los conocimientos sobre los tipos de maltrato, obteniéndose una mayor ocurrencia de resultados aceptables $(60,1 \%)$ para el maltrato psicológico, buenos $(52,4 \%)$ para el abuso sexual y buenos $(70,1 \%)$ para la identifi- 
cación de los indicadores diagnósticos de maltrato (tabla 2). de atención implementado (79,7 \%; IC95 \%: 74,1$85,2)$.

\begin{tabular}{lccc}
\hline Nivel de conocimientos con respecto a... & $\begin{array}{c}\text { Frecuencia } \\
\text { (n=208) }\end{array}$ & $\begin{array}{c}\text { Porcentaje } \\
\text { (\%) }\end{array}$ & IC 95\% \\
\hline Maltrato psicológico & 58 & 27,8 & $21,7-34,0$ \\
Deficiente & 125 & 60,1 & $53,4-66,8$ \\
Aceptable & 25 & 12,0 & $7,6-16,5$ \\
Bueno & & & \\
Abuso sexual & 33 & 15,8 & $10,8-20,9$ \\
Deficiente & 66 & 31,7 & $25,3-38,1$ \\
Aceptable & 109 & 52,4 & $45,6-59,2$ \\
Bueno & & & \\
Indicadores y diagnóstico de maltrato físico & 19 & 9,1 & $5,2-13,1$ \\
Deficiente & 43 & 20,6 & $15,1-26,2$ \\
Aceptable & 146 & 70,1 & $63,9-76,5$ \\
Bueno & &
\end{tabular}

Tabla 2. Nivel de conocimientos con relación al tipo de maltrato infantil en estudiantes de una Facultad de Odontología de Cartagena, Colombia

Acerca de la responsabilidad del odontólogo frente al maltrato infantil, la mayoría de los sujetos de estudio piensan que se debe denunciar a las autoridades competentes, el $73 \%$ conoce dónde se deben hacer las denuncias ante las comisarías de familia o el Instituto de Bienestar Infantil, y frente a la obligación de reporte de la sospecha de maltrato infantil, los estudiantes en un $96 \%$ afirmaron que es obligatorio hacerlo.

Así, también se indagaron por categorías en cuanto a la identificación de lesiones relacionadas con abuso infantil desde la consulta odontológica, obteniéndose los mayores porcentajes en: presencia de moretones en las mejillas (94,2\%), quemaduras en forma de objetos $(90,8 \%)$, repetidas lesiones de avulsión y fractura dental (89,4\%), comportamiento seductor hacia los demás $(65,8 \%)$. De igual forma, un 98,5 \% (IC95 \%: 96,9-100) reconoce la existencia de una relación entre el descuido oral y presencia de abandono físico.

Sin embargo, el 57,6 \% (IC95 \%: 50,9-64,4) de los encuestados consideran que aún no poseen una buena ilustración en el tema, no se sienten preparados para detectar maltrato un 55,7 \% (IC $95 \%$ : $48,9-62,5)$ y por ende la mayoría considera que el tema merece mayor entrenamiento (95\%; IC 95 \%: 92,8-98,4), no conocen protocolos de atención al niño maltratado $(82,6 \%$; IC $95 \%$ : 77,5-87,8) y afirman que en su facultad no existe un protocolo

\section{Actitudes}

Al evaluar el nivel de actitud frente a la problemática de maltrato infantil, se obtuvo que la mayoría (200 estudiantes) presentaron una actitud favorable frente al manejo del maltrato infantil $(96,1 \%$; IC95 \%: 93,5-98,7). En cuanto a la conducta que tomaría si su paciente está siendo maltratado, más de la mitad de los estudiantes hablaría con el docente; ante un caso de negligencia odontológica, la mayoría de los encuestados afirma que lo anotaría en la historia clínica y lo reportaría. Por otra parte, frente a la actitud que tomarían si su paciente es maltratado en la consulta odontológica, los estudiantes contestaron que llamarían la atención al padre o comunicarían al docente con porcentajes similares en ambas respuestas (tabla 3).

\section{Prácticas}

De los 42 estudiantes que reportaron haber tenido alguna sospecha durante su práctica formativa, 15 $(35,7 \%)$ manifestaron que su primera acción ante la sospecha fue comunicarla a su docente, seguida por comentarla a un compañero y guardar silencio. De los que manifestaron guardar silencio la razón que más atribuyeron a su comportamiento fue el no tener la certeza de la sospecha, seguida por el temor a las represarías. Las tres cuartas partes de los estudiantes que manifestaron haber tenido una sospecha de maltrato durante sus prácticas formación no la dejaron registrada en la historia clínica (tabla 4). 


\begin{tabular}{lccc}
\hline Actitudes frente al maltrato infantil ante... & $\begin{array}{c}\text { Frecuencia } \\
(\mathbf{n = 2 0 8 )}\end{array}$ & $\begin{array}{c}\text { Porcentaje } \\
(\mathbf{\%})\end{array}$ & IC 95\% \\
\hline Paciente que está siendo maltratado & & & \\
No hace ni dice nada & 3 & 1,4 & $0,2-3,0$ \\
Hablaría con la familia del niño & 38 & 18,2 & $12,9-23,5$ \\
Confirmaría con vecinos y denunciaría & 28 & 13,4 & $8,7-18,1$ \\
Comentaría al profesor y haría lo que le dijera & 137 & 65,8 & $59,3-72,3$ \\
Otra actitud & 2 & 0,9 & $0,3-2,2$ \\
Un caso de abandono & & & \\
Lo anotaría en la historia clínica y denunciaría al & 142 & 68,2 & $61,8-74,6$ \\
docente & 56 & 26,9 & $20,8-33,0$ \\
Aconsejaría al padre con seguimiento cercano & 8 & 3,8 & $1,2-6,4$ \\
Sólo terminaría los requisitos de la clínica & 2 & 0,9 & $0,3-2,2$ \\
Conversaría con el padre sin seguimiento & & & \\
Paciente al que maltratan en su consulta & 4 & 1,9 & $0,1-3,8$ \\
No hace ni dice nada & 104 & 50,0 & $43,1-56,8$ \\
Reprende al padre & 83 & 39,9 & $33,1-46,6$ \\
Llama al docente & 14 & 6,7 & $3,2-10,1$ \\
Llama a las autoridades y denuncia & 3 & 1,4 & $0,2-3,0$ \\
Otra actitud (saca al padre, aconseja al padre) & & & \\
Niño maltratado por el hermano & 1 & 0,4 & $0,4-1,4$ \\
No hace ni dice nada & 33 & 15,8 & $10,8-20,8$ \\
Aconseja al hermano & 172 & 82,6 & $77,5-87,8$ \\
Habla con la familia del niño & 2 & 0,9 & $0,3-2,2$ \\
Otra actitud & & & \\
\hline
\end{tabular}

Tabla 3. Actitudes frente al maltrato infantil en estudiantes de una Facultad de Odontología de Cartagena, Colombia

La tabla 5 muestra los hallazgos obtenidos al relacionar la sospecha de maltrato infantil con las variables sociodemográficas, conocimientos y actitudes, encontrándose solo diferencias estadísticamente significativas con el semestre cursado $(p=0,008)$, siendo los que más sospecharon los estudiantes de semestres avanzados (octavo a décimo).

\section{DISCUSIÓN}

En este estudio se observó que el nivel de conocimiento frente al maltrato infantil fue adecuado, resaltándose la importancia del reporte del mismo, aunque más de la mitad de los estudiantes afirma no estar lo suficientemente preparado para diagnosticar el MI en su práctica diaria, a diferencia de lo reportado por Al-Jundi et al. ${ }^{16}$, en cuyo trabajo el nivel de conocimiento en los estudiantes de odontología de Jordania fue escaso, aludiendo poca preparación en el tema aunque éstos conocían de la importancia de diagnosticar adecuadamente el maltrato infantil.

Estudios realizados en Turquía en médicos de Atención Primaria sobre el conocimiento y actitud del $\mathrm{Ml}$ concluyeron que eran inadecuados para el diagnóstico y la notificación del $\mathrm{MI}^{17}$. A diferencia de lo reportado por Fierro et al. ${ }^{1}$ en odontólogos de Chile, donde las conocimientos sobre MI fueron satisfactorios, sin embargo, los odontólogos generales no demostraron estar preparados para identificar el Ml. Pires et al. ${ }^{18}$, en Brasil, evaluaron a pediatras sobre el grado de conocimientos de $\mathrm{MI}$, concluyendo que, sin tener estudios específicos sobre el tema, el grado de conocimiento de éstos fue suficiente.

Jouriles et al. ${ }^{19}$, al evaluar y comparar los conocimientos sobre la identificación y el reconocimiento 


\begin{tabular}{lccc}
\hline Prácticas frente a sospecha de maltrato... & $\begin{array}{c}\text { Frecuencia } \\
\text { (n=208) }\end{array}$ & $\begin{array}{c}\text { Porcentaje } \\
\text { (\%) }\end{array}$ & IC 95\% \\
\hline Primera actitud ante la sospecha & & & \\
Hablar con un compañero & 10 & 4,8 & $1,8-7,7$ \\
Hablar con su docente & 15 & 7,2 & $3,6-10,7$ \\
Hablar con los padres del menor & 6 & 2,8 & $0,5-5,1$ \\
Buscar a un pediatra & 1 & 0,4 & $0,4-1,4$ \\
Quedarse callado & 10 & 4,8 & $1,8-7,7$ \\
No aplica & 166 & 79,8 & $74,3-85,3$ \\
Razón por la que se quedó callado & & & \\
No estar seguro del diagnóstico & 4 & 1,9 & $0,1-3,8$ \\
No saber dónde recurrir & 2 & 0,9 & $0,3-2,2$ \\
Miedo a represalias en su contra & 3 & 1,4 & $0,2-3,0$ \\
Miedo a perder el paciente & 1 & 0,4 & $0,4-1,4$ \\
No aplica & 198 & 95,1 & $92,2-98,1$ \\
Consignación de la sospecha en la historia clínica & & & \\
Sí & 10 & 4,8 & $1,8-7,7$ \\
No & 32 & 15,3 & $10,4-20,3$ \\
No aplica & 166 & 79,8 & $74,3-85,3$ \\
Indicios para la sospecha de maltrato & & & \\
Trauma físico del menor & 12 & 5,7 & $2,5-8,9$ \\
Comportamiento temeroso & 13 & 6,2 & $2,9-9,5$ \\
Intento del niño por ocultar lesiones & 1 & 0,4 & $0,4-1,4$ \\
Lesión a nivel cervical o facial & 7 & 3,3 & $1,7-6,0$ \\
Descuido como desaseo, malnutrición & 24 & 11,5 & $7,1-15,9$ \\
Todas las anteriores & 1 & 0,4 & $0,4-1,4$ \\
Otro indicio & 2 & 0,9 & $0,3-2,2$ \\
No aplica & 166 & 79,8 & $74,3-85,3$ \\
Pregunta por conductas sospechosas de maltrato & infantil a los & padres del menor \\
Sí & 17 & 8,1 & $4,4-11,9$ \\
No & 25 & 12,0 & $7,5-16,4$ \\
No aplica & 79,8 & $74,3-85,3$ \\
\hline
\end{tabular}

Tabla 4. Prácticas frente al maltrato infantil en estudiantes de una Facultad de Odontología de Cartagena, Colombia

del $\mathrm{Ml}$ entre médicos, odontólogos y enfermeras en Irlanda del Norte, se encontraron que los odontólogos presentaron las puntuaciones más bajas y además se destacaron por su falta de voluntad en denunciarlos. En este estudio, si bien es cierto que los estudiantes mostraron unos conocimientos y actitudes favorables hacia la importancia y la necesidad del reporte de los casos de MI, se observa una incongruencia con las prácticas, ya que las tres cuartas partes de los estudiantes que alguna vez sospecharon no lo consignaron en la historia clínica ni lo reportaron. Estos resultados son similares a los de otros estudios ${ }^{1,20}$, como el realizado en Dinamarca con odontólogos e higienistas dentales, donde solo el 33,9 \% denunció sus sospechas de $\mathrm{MI}$ a los servicios sociales, no cumpliendo así suficientemente con su rol de protector infantil ${ }^{20}$.

Cuando un odontólogo o estudiante durante su práctica sospecha que una lesión no es de etiología accidental tiene la obligación de documentar 


\begin{tabular}{|c|c|c|c|c|c|}
\hline \multirow{3}{*}{ Factores asociados } & \multicolumn{4}{|c|}{ Sospecha de maltrato infantil } & \multirow[b]{3}{*}{ Valor de $\mathrm{p}$} \\
\hline & \multicolumn{2}{|c|}{ No } & \multicolumn{2}{|c|}{ Sí } & \\
\hline & Frecuencia & Porcentaje & Frecuencia & Porcentaje & \\
\hline \multicolumn{6}{|c|}{ Edad por ciclo individual } \\
\hline $\begin{array}{l}\text { Adolescente } \\
\text { (10-19 años) }\end{array}$ & 24 & 88,8 & 3 & 11,1 & \\
\hline $\begin{array}{l}\text { Adulto joven } \\
\text { (20-44 años) }\end{array}$ & 142 & 78,4 & 39 & 21,5 & 0,2 \\
\hline \multicolumn{6}{|l|}{ Sexo } \\
\hline Mujer & 107 & 80,4 & 26 & 19,5 & \\
\hline Varón & 59 & 78,6 & 16 & 21,3 & 0,7 \\
\hline \multicolumn{6}{|l|}{ Estratificación social } \\
\hline Bajo/medio-bajo (1-3) & 140 & 78,2 & 39 & 21,7 & \\
\hline Medio-alto/alto (4-6) & 26 & 89,6 & 3 & 10,3 & 0,15 \\
\hline \multicolumn{6}{|l|}{ Semestre } \\
\hline Sexto a séptimo & 70 & 95,8 & 3 & 4,1 & \\
\hline Octavo a décimo & 96 & 71,1 & 39 & 28,8 & 0,008 \\
\hline \multicolumn{6}{|c|}{ Nivel de conocimientos } \\
\hline $\begin{array}{l}\text { Deficientes / } \\
\text { Aceptables }\end{array}$ & 75 & 78,9 & 20 & 21,0 & \\
\hline Buenos & 91 & 80,5 & 22 & 19,4 & 0,7 \\
\hline \multicolumn{6}{|c|}{ Conocimientos sobre indicadores de diagnóstico de maltrato infantil } \\
\hline Buenos & 102 & 78,4 & 28 & 21,5 & \\
\hline Deficientes & 61 & 81,3 & 14 & 18,6 & 0,6 \\
\hline \multicolumn{6}{|l|}{ Nivel de actitud } \\
\hline Favorables & 159 & 79,5 & 41 & 20,5 & \\
\hline Desfavorables & 7 & 87,5 & 1 & 12,5 & 0,5 \\
\hline
\end{tabular}

Tabla 5. Relación entre sospecha de maltrato infantil y factores sociodemográficos, conocimientos y actitudes de estudiantes de Odontología de Cartagena, Colombia

y recoger las evidencias de las lesiones, incluyendo documentación escrita (realizando diagramas cuando sea posible y describiendo los hallazgos físicos por su número, tipo, tamaño, ubicación y estado; la sospecha de maltrato infantil debe ser mencionada, con una explicación adecuada que justifique esa sospecha) ${ }^{21}$, fotografías, radiografías y modelos de estudio diagnóstico. Estos hallazgos pueden ser necesarios para ser escrutados por el sistema judicial y los expertos que testimonien en el caso ${ }^{22}$. Sin embargo, pocas veces se cumple con la obligación ético-legal de proteger al más débil. Ello se debe, entre otras razones, al desconocimiento de las leyes específicas, la poca protección del estado a los profesionales implicados en el caso, a experiencias anteriores que no se resolvieron favorablemente o que generaron sanciones para el denunciante, al temor a las represalias y al hostigamiento que pueden sufrir los profesionales, a veces víctimas de un acoso por los abogados, cuya consecuencia es un rechazo a todo lo relacionado con lo legal; por ello, algunos optan por no denunciar ${ }^{23}$, tal como se evidencia en este estudio, donde dentro de las razones que adujeron los estudiantes para guardar silencio ante el reporte fue el no estar seguro del diagnóstico y el temor a las represalias. Caso similar a lo encontrado por Fierro et al. y Pires et al., donde las razones fueron la falta de conocimientos y el temor a sanciones legales al denunciar a un caso de maltrato infantil, respectivamente ${ }^{1,18}$.

Al preguntar dónde se deberían denunciar los casos sospechosos de abuso infantil, la mayoría de 
los estudiantes respondieron correctamente, en contraposición con el estudio de Jordania ${ }^{17}$, en el que casi dos tercios de la población del estudio no sabían dónde hacerlo, al igual que lo reportado en otros estudios con resultados similares entre los estudiantes ${ }^{24,25}$.

En este estudio se reporta una prevalencia importante de casos de sospecha de maltrato infantil identificada por los estudiantes, siendo mayor en los semestres avanzados ( $8^{\circ}-10^{\circ}$ semestre); posiblemente esto se deba a que al aumentar la práctica clínica y trato con los menores, los conocimientos sobre el tema pasan de lo teórico a lo práctico ganando más experiencia y mayor posibilidad de identificar situaciones sospechosas en sus paciente. Aunque, desafortunadamente, estas sospechas no se están denunciando ni consignando en la historia clínica.

Dada la alta prevalencia de abuso de menores y negligencia en Colombia y el alto porcentaje de las heridas bucales y faciales como consecuencia del maltrato infantil ${ }^{26}$, es crucial para los profesionales de la salud estar bien informados y capacitados sobre el abuso/negligencia en menores; el futuro odontólogo, por lo tanto, tiene un papel esencial en la detección primaria y el control de los malos tratos; además, están obligados por ley a denunciar los presuntos casos de malos tratos infantiles ante las autoridades protectoras de menores ${ }^{27}$. Sin embargo, no es responsabilidad del odontólogo hacer el diagnóstico de un niño maltratado, porque no posee los conocimientos médicos ni psiquiátricos para poder establecer diferencias entre los signos de maltrato y algunas condiciones o lesiones que surgen por otras enfermedades. Pero sí puede, durante el examen clínico, alertarse y sospechar la posibilidad de estar frente a un niño maltratado físicamente, como resultado de las heridas o lesiones que afectan a las estructuras orales ${ }^{14}$.

Los estudiantes de la Facultad de Odontología de la Universidad de Cartagena poseen conocimientos y actitudes adecuadas para detectar las lesiones orales sospechosas del maltrato infantil, pero necesitan más información y motivación que les brinde seguridad en el momento de sospechar y denunciar los posibles casos de maltrato infantil y la existencia de protocolos claros de reporte y su socialización. Es pertinente, dada la importancia de la problemática a nivel mundial, que se incluya en los planes de estudios de los programas de salud, y particularmente de odontología, para una mayor preparación de los estudiantes y futuros profesionales; de igual forma, se recomienda la elaboración de protocolos de manejo de los casos de niños maltratados o abusados en el interior de las clínicas odontológicas.

\section{BIBLIOGRAFÍA}

1. Fierro C, Salazar E, Ruiz L, Luengo I, Pérez A. Maltrato infantil: actitud y conocimiento de odontólogos en Concepción, Chile. Int J Odontostomat 2012; 6 (1): 105-10.

2. Morante CA, Kanashiro CA. El odontólogo frente al maltrato infaltil. Rev estomatol Herediana. 2009; 19 (1): 50-4.

3. Manea S, Favero GA, Stellini E, Romoli L, Mazzucato M, Facchin P. Dentists' perceptions, attitudes, knowledge and experience about child abuse and neglect in northeast Italy. J Clin Pediatr Dent. 2007; 32 (1):19-25.

4. Naidoo S. A profile of the oro-facial injuries in child physical abuse at a children's Hospital. Child Abuse Negl. 2000; 24 (4): 521-34.

5. Cairns AM, Mok JY, Welbury RR. The dental practioner and child protection in Scotland. Br Dent J. 2005; 199 (8): 51720.

6. Cavalcanti AL. Prevalence and characteristics of injures to the head and orofacial region in physically abused children and adolescents - a retrospective study in a city of the Northeast or Brazil. Dent Traumatol. 2010; 26 (2): 149-53.

7. Bsoul SA, Flint DJ, Dove SB, Senn DR, Alder ME. Reporting of child abuse: a follow-up survey of Texas dentists. Pediatr Dent. 2003; 25 (6): 541-5.

8. Jessee SA. Risk factors as determinants of dental neglect in children. ASDC J Dent Child. 1998; 65 (1): 17-20.

9. John V, Messer LB, Arora R, Fung S, Hatzis E, Nguyen T et al. Child abuse and dentistry: a study of knowledge and attitudes among dentists in Victoria, Australia. Aust Dent $\mathrm{J}$. 1999; 44 (4): 259-67.

10. Harris JC, Elcock C, Sidebotham PD, Welbur RR. Safeguarding children in dentistry: 1 . Child protection training, experience and practice of dental professionals with an interest in paediatric dentistry. Br Dent J. 2009: 206 (8): 409-14.

11. Sinha $S$, Acharya $P$, Jafar $H$, Bower EJ, Harrison VE, Newton JT. The management of abuse: A resource manual for the dental team. London: Stephen Hancocks Ltd; 2005. p. 10-34

12. Sonbol HN, Abu-Ghazaleh S, Rajab LD, Baqain ZH, Saman R, Al-Bitar ZB. Knowledge, educational experiences and attitudes towards child abuse amongst Jordanian dentists. Eur J Dent Educ. 2012; 16 (1): 158-65.

13. Saliba O, Garbin CA, Garbin AJ, Dossi AP. Responsabilidade do professional de saúde sobre a notificação de casos de violência doméstica. Rev Saúde Pública. 2007; 41 (3): 472-7.

14. Ferro $M$, Maldonado $A$, Montiel $Y$, Rivas $M$. Implicaciones psicológicas del paciente odontológico con maltrato infantil. Revista Latinoamericana de Ortodoncia y Odontopediatría "Ortodoncia.ws" edición electrónica enero 2010. Disponible en: www.ortodoncia.ws. Consultada 11/03/2014.

15. Adair SM, Wray IA, Hanes CM, Sams DR, Yasrebi S, Russell CM. Perceptions associated with dentists' decisions to report hypothetical cases of child maltreatment. Pediatr Dent. 1997; 19 (8): 461-5.

16. Al-Jundi $\mathrm{SH}$, Zawaideh FI, Al-Rawi MH. Jordanian dental students' knowledge and attitudes in regard to child physical abuse. J Dent Educ. 2010; 74 (10): 1159-65. 
17. Açik $Y$, Deveci SE, Oral R. Level of knowledge and attitude of primary care physicians in Eastern Anatolian cities in relation to child abuse and neglect. Prev Med. 2004; 39 (4): 791-7.

18. Pires JM, Zubaran M, Vieira EM, Nava TR, Feldens L, Castilhos $\mathrm{K}$ et al. Barreiras, para a notificação pelo pediatra, de maus-tratos infantis. Rev Bras Saude Mater Infant. 2005; 5 (1): 103-8.

19. Jouriles EN, McDonald R, Slep AM, Heyman RE, Garrido E. Child abuse in the context of domestic violence: prevalence, explanations, and practice implications. Violence Vict. 2008; 23 (2): 221-35.

20. Uldum B, Christensen HN, Welbury R, Poulsen S. Danish dentists' and dental hygienists' knowledge of and experience with suspicion of child abuse or neglect. Int $\mathrm{J}$ Paediatr Dent. 2011; 20 (5): 361-5.

21. Sheffield M. Safeguarding children: the case for mandatory training. Community Pract. 2008; 81 (7): 27-30.

22. Medrano G, Perona G. Maltrato infantil: una realidad muy cercana, ¿cómo debemos actuar los odontólogos? Odontol Pediatr. 2010; 9 (1): 78-94.

23. Averbuj G, Bozzalla L, Marina M, Tarantino G, Zaritzky G. Violencia y escuela. Propuestas para comprender y actuar. Buenos Aires: Aique; 2007.
24. Owais Al, Qudeimat MA, Qodceih S. Dentists' involvement in identification and reporting of child physical abuse: Jordan as a case study. Int J Paediatr Dent. 2009; 19 (4): 2916.

25. Thomas JE, Straffon L, Inglehart MR. Child abuse and neglect: dental and dental hygiene students' educational experiences and knowledge. J Dent Educ. 2006; 70 (5): 558-65.

26. Unicef - Fondo de las Naciones Unidas para la infancia. Oficina de Área para Colombia y Venezuela. La niñez de Colombia en cifras [Internet]. Unicef; 2002 [consultada 11/03/2014]. Disponible en: http://www.unicef.org/colombia/pdf/cifras.pdf

27. Ministerio de Salud de Colombia. Resolución 412 del 2000 (25 de febrero), por la cual se establecen las actividades, procedimientos e intervenciones de demanda inducida y obligatorio cumplimiento y se adoptan las normas técnicas y guías de atención para el desarrollo de las acciones de protección específica y detección temprana y las enfermedades de interés en salud pública. 